\title{
Levantamento florístico de um brejo-herbáceo localizado na restinga de Morada do Sol, município de Vila Velha, Espírito Santo, Brasil
}

Floristic survey of a sedge-swamp located in the Morada do Sol restinga, Vila Velha, Espirito Santo, Brazil

\author{
Rodrigo Theófilo Valadares ${ }^{1,4}$, Fabiana Baleeiro Coelho de Souza ${ }^{2}$, Nathália Góes Duarte de Castro², \\ Ana Lúcia Soares da Silva Peres ${ }^{2}$, Solange Zanotti Schneider ${ }^{2} \&$ Márcio Lacerda Lopes Martins $^{3}$
}

\begin{abstract}
Resumo
Este trabalho apresenta a composição florística de um brejo-herbáceo localizado em Morada do Sol, Vila Velha, Espírito Santo. As coletas foram realizadas através de caminhadas ao acaso, sendo coletados ramos férteis de angiospermas e samambaias. Foram registradas 125 espécies distribuídas em 56 famílias. A família Asteraceae foi a que apresentou maior riqueza específica com 13 espécies, seguida por Cyperaceae com 12, Fabaceae com nove, Rubiaceae com oito e Poaceae com sete espécies. Das espécies encontradas Melanopsidium nigrum Colla (Rubiaceae) encontra-se na lista oficial de espécies ameaçadas de extinção e 29 são citadas pela primeira vez para vegetação de restinga do Espírito Santo. A presença de 28 espécies invasoras provém de alterações provocadas pela diminuição da área inundável através de aterro e queimadas intencionais com intervalos curtos, que proporcionam alterações florísticas e fisionômicas neste ecossistema.

Palavras-chave: riqueza, conservação, macrófitas, restingas.
\end{abstract}

\begin{abstract}
This paper presents the floristic composition of a sedge- swamp located in Morada do Sol, Vila Velha, Espirito Santo. Samples were collected through random walks, these being fertile branches of angiosperms and ferns. We recorded 125 species in 56 families. The Asteraceae family showed highest species richness with 13 species, followed by Cyperaceae with 12, Fabaceae 9, Rubiaceae and Poaceae with 8 and 7 species respectively. Melanopsidium nigrum Colla is on the official list of endangered species and 29 other species are first citations for the salt marsh vegetation of Espirito Santo. The presence of 28 invasive species is due to changes caused by the decrease in the flooded area by intentional landfill and burning at short intervals, which promote floristic and physiognomic changes in the ecosystem.
\end{abstract}

Key words: richness, conservation, macrophytes, sandy coastal plains.

\section{Introdução}

Os brejos constituem uma das diversas fitofisionomias ocorrentes nas restingas brasileiras. Dentre as formações que compõem as restingas, três delas constituem os brejos: herbácea inundada, herbácea inundável e arbustiva inundável (Pereira 2003).

Caracterizados por alagamento sazonal e ocupados principalmente por representantes das famílias Poaceae e Cyperaceae, os brejos apresentam-se bem desenvolvidos nos litorais do Rio Grande do Sul e ao norte do estado do Rio de Janeiro (Lacerda et al. 1993). Trata-se de um ecossistema no qual as espécies possuem a capacidade de resistir à submersão permanente ou sazonal (Amaral et al. 2008).

A vegetação deste tipo de ambiente desenvolve-se sobre um solo orgânico de espessura variável sobre um horizonte de areia. A alta

\footnotetext{
'Universidade Federal do Espírito Santo, Herbário VIES, Av. Fernando Ferrari 514, Goiabeiras, 29075-910,Vitória, ES, Brasil.

${ }^{2}$ Centro Universitário Vila Velha, Depto. Ciências Biológicas, R. José Dantas de Melo 21, Boa Vista, 29102-770, Vila Velha, ES, Brasil.

${ }^{3}$ Universidade Federal do Recôncavo da Bahia, R. Rui Barbosa 710, Cruz das Almas, 44380-000, BA, Brasil.

${ }^{4}$ Autor para correspondência: rodrigotheofilo@yahoo.com.br
} 
produtividade primária e a sazonalidade bem marcada, dependente das condições do alagamento, interferem diretamente na composição e distribuição da vegetação constituinte (Lacerda et al. 1986).

No Espírito Santo os brejos são caracterizados pela descontinuidade ocasionada pela elevação do nível do solo ou por ocupação urbana desordenada. Neste Estado os estudos florísticos nestes ambientes são escassos, destacando-se apenas o trabalho taxonômico com Cyperaceae, realizado por Martins et al. (1999), no qual foram registradas 24 espécies para o Parque Estadual Paulo César Vinha, no município de Guarapari. Neste estudo verificou-se a existência de um novo táxon para a ciência e exclusiva desta formação, Rhynchospora plusquamrobusta Luceño \& M. Martins, o que indica a necessidade de estudos florísticos mais aprofundados com este tipo de formação, para o melhor conhecimento de sua biodiversidade. A dificuldade de acesso e de locomoção neste ambiente pode ser citada como possível causa para a escassez de estudos, porém somente com a realização de mais pesquisas poderão ser fornecidos dados para avaliação, manejo e conservação deste ecossistema, ampliando os dados florísticos para as restingas do Espírito Santo.
O presente estudo visa contribuir para este aperfeiçoamento, apresentando uma listagem florística para a formação brejo-herbáceo da restinga de Morada do Sol, município de Vila Velha, ES.

\section{Material e Métodos \\ Área de estudo}

A área de estudo localiza-se a leste da Rodovia do Sol ES-060, em um remanescente de restinga com área total de 123,55 ha, situado entre as coordenadas $20^{\circ} 27^{\prime} 39,44^{\prime}$ ' $S$ e $40^{\circ} 20^{\prime} 23,41^{\prime \prime} \mathrm{W}$, correspondente ao município de Vila Velha, ES (Fig. 1). A área que corresponde ao brejo subdivide-se em quatro áreas isoladas devido aterramento, que juntas totalizam $26,52 \mathrm{ha}$, sem conexão com o mar. O corpo d'água apresenta coloração amarronzada, devido à rica presença de substâncias húmicas, comuns para este ambiente em restingas. A profundidade máxima atinge $1,5 \mathrm{~m}$ nos meses mais chuvosos entre dezembro e janeiro, secando completamente nos meses de junho e julho. O clima predominante na região é do tipo AW tropical segundo a classificação de Köppen, com verão quente e chuvoso e inverno seco com precipitação pluviométrica anual média em torno de $1.300 \mathrm{~mm}$, sendo mais abundante no verão (Magnago et al. 2007).

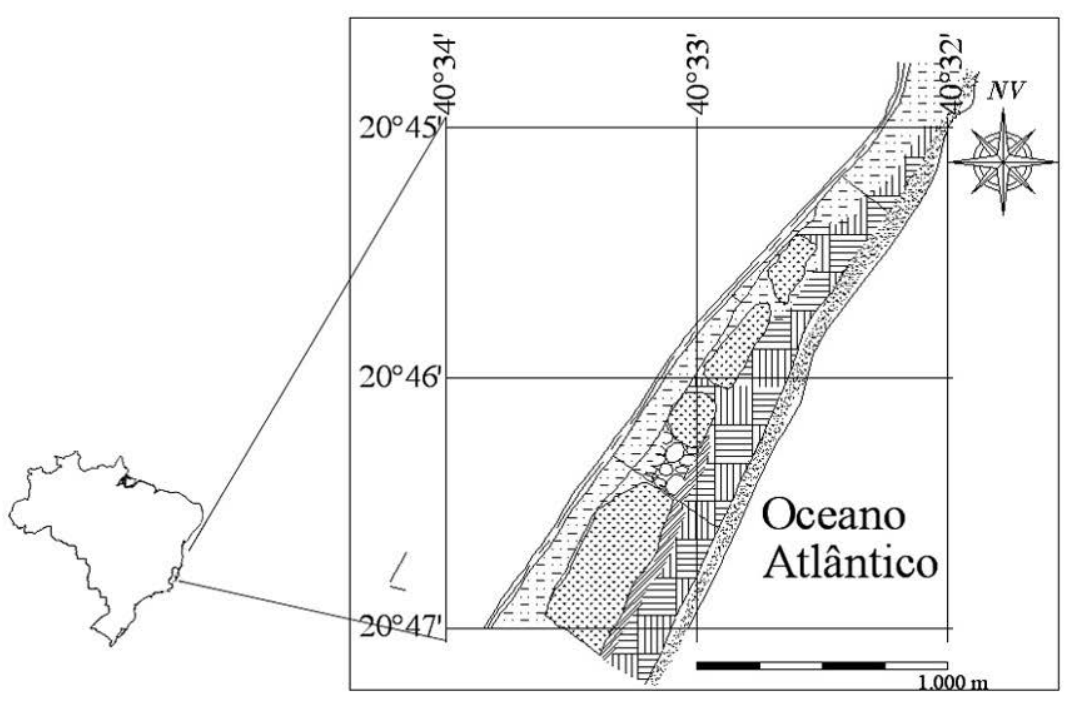

\section{Convenções}

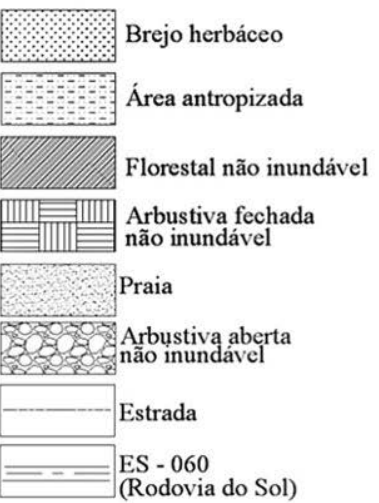

Figura 1- Formações vegetais da restinga de Morada do Sol. Fonte: Modificado de Magnago et al. (2007). Figure 1 - Vegetation of the restinga of Morada do Sol. Source: Modified from Magnago et al. (2007). 


\section{Coleta e análise do material botânico}

Foram realizadas coletas mensais durante 12 meses consecutivos a partir de agosto de 2007, coletando-se ramos férteis de vegetais vasculares através de trilhas pré-existentes e/ou por meio de caminhadas sem orientação pré-estabelecida.

As coletas seguiram os procedimentos descritos por Fidalgo \& Bononi (1989), sendo todo o material coletado depositado no Herbário VIES da Universidade Federal do Espírito Santo.

A identificação do material botânico foi realizada através de bibliografia especializada (Barroso 1991a,b; Barroso et al. 2002; Souza \& Lorenzi 2005; Amaral et al. 2008; Lorenzi 2008), ou envio de exsicatas a especialistas. As espécies consideradas invasoras foram determinadas conforme Lorenzi (2008). As Angiospermas são apresentadas de acordo com a classificação proposta pelo APGIII (2009) e as Lycophytas e Monilophytas seguem Christenhusz et al. (2011).

A forma de vida de cada espécie foi adotada apenas para indivíduos férteis e seguiu a terminologia adotada por Pedralli (1990). Para as espécies arbustivas e escandentes de outros ambientes tolerantes a inundação periódica utilizou-se apenas os termos "arbustivo"e "escandente" para o hábito.

\section{Resultados e Discussão}

A formação brejo-herbáceo da restinga de Morada do Sol é caracterizada por apresentar porte predominantemente herbáceo com ocorrência ocasional de espécies arbustivas tolerantes ao período de afloramento do lençol freático. Os brejos são responsáveis pelo armazenamento de água proveniente do excesso de chuvas (Irgang \& Gastal Junior 1996), o que pode contribuir para que áreas urbanas adjacentes, como o bairro Morada do Sol, não sejam inundadas.

Para esta formação, em Morada do Sol, foram encontradas 125 espécies distribuídas em 56 famílias (Tab. 1), sendo 50 famílias correspondentes às Angiospermas, cinco famílias ao grupo das Monilophytas e uma Lycophyta. Alguns táxons não puderam ser identificados a nível específico, por terem sido coletadas apenas em estágio de frutificação, totalizando dez espécies. As famílias mais representativas foram Asteraceae, com 13 espécies, seguida por Cyperaceae (12), Fabaceae (9), Rubiaceae (8) e Poaceae com sete espécies (Fig. 2). As famílias que foram representadas por apenas um táxon totalizaram $25 \%$. Considerando a listagem florística produzida por Pereira \& Araújo (2000) para as restingas

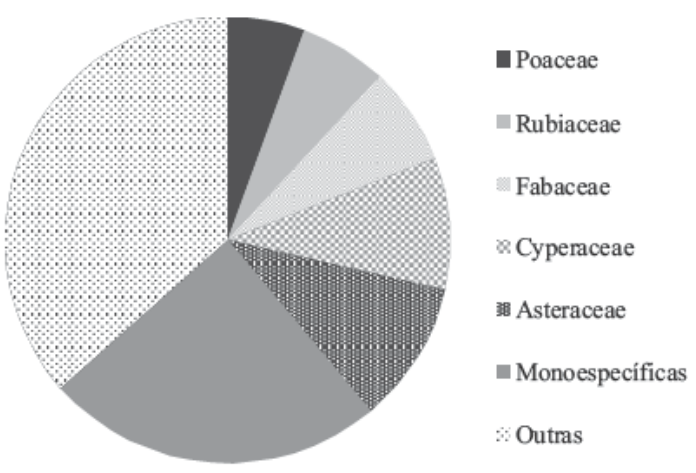

Figura 2 - Famílias com maior e menor riqueza de espécies vasculares do brejo-herbáceo de Morada do Sol, Vila Velha, ES.

Figure 2 - Families with higher and lower species richness of vascular sedge marsh of Morada do Sol, Vila Velha, ES.

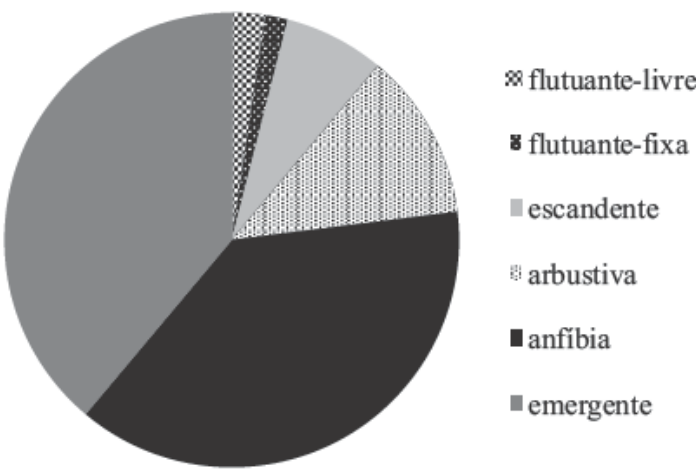

Figura 3 - Formas biológicas das espécies vasculares do brejo-herbáceo de Morada do Sol, Vila Velha, ES. Figure 3-Biological forms of species of vascular sedge marsh from Morada do Sol, Vila Velha, ES. Following the sequence as shown in the figure: fixed floating, free floating, climbing, shrubby, amphibious, and emergent.

do estado do Espírito Santo e Riode Janeiro, 29 táxons do presente estudo são novas ocorrências para as restingas do estado do Espírito Santo.

A formade vida predominante para os indivíduos férteis de Moradado Sol foi a emergente com49espécies, seguida pela anfíbia com 48, flutuante-livre com trêse flutuante-fixa com duas espécies (Fig. 3). As espécies arbustivas e escandentes com tolerância a inundação estiveram representadas por 15 e nove espécies, respectivamente. Em ecossistemas aquáticos temporários não existe uma única forma de vida entre os indivíduos devido à periodicidade de inundação. Mesmo espécies flutuantes podem ser encontradas como anfíbias desenvolvendo-se em solos úmidos, como Nymphoides indica (Bove et al. 2003). 
Tabela 1 - Famílias, gêneros e espécies ocorrentes na formação brejo-herbáceo da restinga de Morada do Sol, Vila Velha, ES. ${ }^{1}$ ameaçada de extinção IBAMA (2008), ${ }^{2}$ espécie invasora, ${ }^{3}$ nova ocorrência para as restingas do Espírito Santo.

Table 1 - Families, genera and species of the sedge marsh formation of the restinga Morada do Sol, Vila Velha, ES. ${ }^{1}$ endangered IBAMA (2008), ${ }^{2}$ invasive species, ${ }^{3}$ new occurrence for the restinga of the Espirito Santo.

\begin{tabular}{|c|c|c|c|}
\hline Família & Nome científico & $\begin{array}{l}\mathbf{N}^{\circ} \\
\text { coletor }\end{array}$ & $\begin{array}{l}\text { Forma } \\
\text { de vida }\end{array}$ \\
\hline Alismataceae & Sagittaria lancifolia $\mathrm{L}$. & B-133 & anfíbia \\
\hline Anacardiaceae & Schinus terebinthifolius Raddi & B-35 & arbustiva \\
\hline Apocynaceae & $\begin{array}{l}\text { Oxypetalum alpinum (Vell.) Fontella \& E.A.Schwarz } \\
\text { Temnadenia violacea (Vell.) Miers }\end{array}$ & $\begin{array}{l}\text { B-19 } \\
\text { B-108 }\end{array}$ & $\begin{array}{l}\text { escandente } \\
\text { escandente }\end{array}$ \\
\hline Asteraceae & $\begin{array}{l}\text { Achyrocline satureioides (Lam.) DC. }{ }^{2} \\
\text { Ageratum conyzoides (L.)L. }{ }^{2} \\
\text { Ageratum sp. } \\
\text { Baccharis genistelloides subsp. crispa (Spreng.) Joch.Müll. } \\
\text { Chromolaena odorata (L.) R.M.King \& H.Rob. }{ }^{3} \\
\text { Cyrtocymura scorpioides (Lam.) H.Rob. }{ }^{2} \\
\text { Eclipta prostrata } \text { (L.) L. }{ }^{2} \\
\text { Emilia fosbergii Nicolson }{ }^{2} \\
\text { Ethulia conyzoides } \text { L.f. }^{3} \\
\text { Mikania micrantha } \text { Kunth }{ }^{3} \\
\text { Sonchus oleraceus } \text { (L.) L. }{ }^{2} \\
\text { Sphagneticola trilobata (L.) Pruski }{ }^{2} \\
\text { Lepidaploa rufogrisea (A. St.-Hil.) H.Rob. }\end{array}$ & $\begin{array}{l}\text { B-125 } \\
\text { B-101 } \\
\text { B-128 } \\
\text { B-65 } \\
\text { B-39 } \\
\text { B-38 } \\
\text { B-135 } \\
\text { B-136 } \\
\text { B-37 } \\
\text { B-3 } \\
\text { B-131 } \\
\text { B-134 } \\
\text { B-60 }\end{array}$ & $\begin{array}{l}\text { emergente } \\
\text { emergente } \\
\text { anfibia } \\
\text { emergente } \\
\text { emergente } \\
\text { emergente } \\
\text { emergente } \\
\text { emergente } \\
\text { emergente } \\
\text { emergente } \\
\text { emergente } \\
\text { anfibia } \\
\text { emergente }\end{array}$ \\
\hline Begoniaceae & Begonia fischeri Schrank ${ }^{3}$ & B-83 & anfibia \\
\hline Bignoniaceae & Lundia cordata (Vell.) DC. & B-109 & escandente \\
\hline Boraginaceae & Cordia curassavica (Jacq.) Roem. \& Schult. ${ }^{3}$ & B-110 & anfibia \\
\hline Burmanniaceae & Burmannia capitata (Walter ex J.F.Gmel.) Mart. ${ }^{3}$ & B-28 & anfibia \\
\hline Cactaceae & Cereus fernambucensis Lem. & B-1 & anfibia \\
\hline Celastraceae & Maytenus obtusifolia Mart. & B-117 & arbustiva \\
\hline Clusiaceae & Garcinia brasiliensis Mart. & B-114 & arbustiva \\
\hline Commelinaceae & Commelina virginica L. ${ }^{3}$ & B-49 & anfibia \\
\hline Convolvulaceae & $\begin{array}{l}\text { Cuscuta racemosa } \text { Mart. }^{2} \\
\text { Ipomoea squamosa } \text { Choisy }^{3}\end{array}$ & $\begin{array}{l}\text { B-82 } \\
\text { B-26 }\end{array}$ & $\begin{array}{l}\text { escandente } \\
\text { escandente }\end{array}$ \\
\hline Cyperaceae & $\begin{array}{l}\text { Fimbristylis bahiensis Steud. } \\
\text { Cyperus haspan L. } \\
\text { Cyperus ligularis L. }{ }^{3} \\
\text { Cyperus sesquiflorus (Torr.) Mattf. \& Kük. } \\
\text { Eleocharis geniculata (L.) Roem. \& Schult. } \\
\text { Fuirena umbellata } \text { Rottb. }{ }^{2} \\
\text { Lagenocarpus rigidus (Kunth) Nees. }_{\text {Pycreus polystachyos (Rottb.) P.Beauv. }}^{2} \\
\text { Rhynchospora gigantea Link } \\
\text { Rhynchospora holoschoenoides (Rich.) Herter } \\
\text { Rhynchospora tenuis Link } \\
\text { Scleria hirtella Sw. }\end{array}$ & $\begin{array}{l}\text { B-62 } \\
\text { B-42 } \\
\text { B-98 } \\
\text { B-99 } \\
\text { B-61 } \\
\text { B-43 } \\
\text { B-63 } \\
\text { B-64 } \\
\text { B-97 } \\
\text { B-41 } \\
\text { B-46 } \\
\text { B-126 }\end{array}$ & $\begin{array}{l}\text { anfibia } \\
\text { anfibia } \\
\text { emergente } \\
\text { emergente } \\
\text { emergente } \\
\text { emergente } \\
\text { emergente } \\
\text { emergente } \\
\text { emergente } \\
\text { emergente } \\
\text { emergente } \\
\text { emergente }\end{array}$ \\
\hline Eriocaulaceae & $\begin{array}{l}\text { Paepalanthus tortilis (Bong.) Mart. ex Körn. } \\
\text { Paepalanthus sp. }\end{array}$ & $\begin{array}{l}\text { B-81 } \\
\text { B-69 }\end{array}$ & $\begin{array}{l}\text { emergente } \\
\text { anfibia }\end{array}$ \\
\hline Erythroxylaceae & $\begin{array}{l}\text { Erythroxylum hamigerum O.E.Schulz }{ }^{3} \\
\text { Erythroxylum deciduum A.St.-Hil. }{ }^{3}\end{array}$ & $\begin{array}{l}\text { B-129 } \\
\text { B-80 }\end{array}$ & $\begin{array}{l}\text { emergente } \\
\text { emergente }\end{array}$ \\
\hline
\end{tabular}




\begin{tabular}{|c|c|c|c|}
\hline Família & Nome científico & $\begin{array}{c}\mathbf{N}^{\circ} \\
\text { coletor }\end{array}$ & $\begin{array}{l}\text { Forma } \\
\text { de vida }\end{array}$ \\
\hline Euphorbiaceae & $\begin{array}{l}\text { Alchornea triplinervia (Spreng.) Müll. Arg. } \\
\text { Croton glandulosus L. } \\
\text { Manihot tripartita (Spreng.) Müll. Arg. }\end{array}$ & $\begin{array}{l}\text { B-18 } \\
\text { B-51 } \\
\text { B-120 }\end{array}$ & $\begin{array}{l}\text { arbustiva } \\
\text { anfibia } \\
\text { anfibia }\end{array}$ \\
\hline Fabaceae & $\begin{array}{l}\text { Centrosema brasilianum (L.)Benth. } \\
\text { Chamaecrista flexuosa }(\mathrm{L} .) \mathrm{Green \textrm {e } ^ { 2 }} \\
\text { Chamaecrista ramosa (Vogel) H.S.Irwin \& Barneby } \\
\text { Crotalaria pallida Aiton }{ }^{2} \\
\text { Desmodium barbatum (L.) Benth. }{ }^{2} \\
\text { Mimosa pigra L. }{ }^{3} \\
\text { Senna appendiculata } \text { (Vogel) Wiersema } \\
\text { Stylosanthes guianensis (Aubl.) Sw. }{ }^{2} \\
\text { Stylosanthes viscosa } \text { Sw. }^{2}\end{array}$ & $\begin{array}{l}\text { B-13 } \\
\text { B-36 } \\
\text { B-113 } \\
\text { B-115 } \\
\text { B-116 } \\
\text { B-122 } \\
\text { B-30 } \\
\text { B-111 } \\
\text { B-47 }\end{array}$ & $\begin{array}{l}\text { emergente } \\
\text { emergente } \\
\text { emergente } \\
\text { emergente } \\
\text { emergente } \\
\text { anfibia } \\
\text { arbustiva } \\
\text { anfibia } \\
\text { anfibia }\end{array}$ \\
\hline Gentianaceae & $\begin{array}{l}\text { Curtia verticillaris (Spreng.) Knobl. } \\
\text { Schultesia } \text { sp. }\end{array}$ & $\begin{array}{l}\text { B-17 } \\
\text { B-24 }\end{array}$ & $\begin{array}{l}\text { anfibia } \\
\text { anfibia }\end{array}$ \\
\hline Lamiaceae & Hyptis brevipes Poit. ${ }^{2}$ & B-20 & anfibia \\
\hline Lentibulariaceae & $\begin{array}{l}\text { Utricularia erectiflora A.St.-Hill. \& Girard }{ }^{3} \\
\text { Utricularia subulata } \text { L. }^{3}\end{array}$ & $\begin{array}{l}\text { B-45 } \\
\text { B-92 }\end{array}$ & $\begin{array}{l}\text { flutuante-livre } \\
\text { flutuante-livre }\end{array}$ \\
\hline Lythraceae & Cuphea flava Spreng. & B-103 & emergente \\
\hline Malpighiaceae & $\begin{array}{l}\text { Byrsonima sericea DC. } \\
\text { Peixotoa hispidula A.Juss }\end{array}$ & $\begin{array}{l}\text { B-75 } \\
\text { B-50 }\end{array}$ & $\begin{array}{l}\text { arbustiva } \\
\text { anfibia }\end{array}$ \\
\hline Malvaceae & $\begin{array}{l}\text { Sida ciliaris } \mathrm{L} . \\
\text { Sida } \mathrm{sp} . \\
\text { Waltheria indica } \mathrm{L}^{2}\end{array}$ & $\begin{array}{l}\text { B-105 } \\
\text { B-112 } \\
\text { B-74 }\end{array}$ & $\begin{array}{l}\text { anfibia } \\
\text { anfibia } \\
\text { emergente }\end{array}$ \\
\hline Melastomataceae & $\begin{array}{l}\text { Acisanthera sp. } \\
\text { Marcetia taxifolia (A. St.-Hil.) DC. } \\
\text { Pterolepis glomerata (Rottb.) Miq. }{ }^{3} \\
\text { Tibouchina urceolaris Cogn. }\end{array}$ & $\begin{array}{l}\text { B-104 } \\
\text { B-121 } \\
\text { B-11 } \\
\text { B-15 }\end{array}$ & $\begin{array}{l}\text { anfibia } \\
\text { anfibia } \\
\text { anfibia } \\
\text { emergente }\end{array}$ \\
\hline Menyanthaceae & Nymphoides indica (L.) Kuntze ${ }^{2}$ & B-71 & flutuante-fixa \\
\hline Myrtaceae & $\begin{array}{l}\text { Calyptranthes brasiliensis Spreng. } \\
\text { Syzygium ovalifolium (Blume) Merr. \& L.M.Perry }\end{array}$ & $\begin{array}{l}\text { B-72 } \\
\text { B-107 }\end{array}$ & $\begin{array}{l}\text { arbustiva } \\
\text { arbustiva }\end{array}$ \\
\hline Nyctaginaceae & $\begin{array}{l}\text { Guapira opposita (Vell.) Reitz } \\
\text { Guapira pernambucensis (Casar.) Lundell }\end{array}$ & $\begin{array}{l}\text { B-118 } \\
\text { B-76 }\end{array}$ & $\begin{array}{l}\text { arbustiva } \\
\text { arbustiva }\end{array}$ \\
\hline Nymphaeaceae & Nymphaea rudgeana G.Mey. ${ }^{3}$ & B-53 & flutuante-fixa \\
\hline Ochnaceae & $\begin{array}{l}\text { Ouratea cuspidata Engl. ex Gilg. } \\
\text { Sauvagesia erecta L. }\end{array}$ & $\begin{array}{l}\text { B-6 } \\
\text { B-33 }\end{array}$ & $\begin{array}{l}\text { emergente } \\
\text { anfibia }\end{array}$ \\
\hline Olacaceae & Heisteria silvianii $\mathrm{Schwacke}^{3}$ & B-85 & arbustiva \\
\hline Onagraceae & $\begin{array}{l}\text { Ludwigia leptocarpa (Nutt.) H.Hara } \\
\text { Ludwigia longifolia (DC.) H.Hara }\end{array}$ & $\begin{array}{l}\text { B-138 } \\
\text { B-67 }\end{array}$ & $\begin{array}{l}\text { anfibia } \\
\text { anfibia }\end{array}$ \\
\hline Orchidaceae & Habenaria parviflora Lindl. & B-87 & anfibia \\
\hline Passifloraceae & Passiflora alata Curtis & B-132 & escandente \\
\hline Peraceae & Pera glabrata (Schott) Poepp. ex Baill. & B-79 & arbustiva \\
\hline Phyllanthaceae & Phyllanthus niruri L.2 & B-86 & anfibia \\
\hline Poaceae & $\begin{array}{l}\text { Andropogon bicornis } \mathrm{L}^{2} \\
\text { Andropogon leucostachyus Kunth. } \\
\text { Eriochrysis cayennensis P.Beauv. } \\
\text { Panicum sp. } \\
\text { Panicum wettsteinii Hack. }\end{array}$ & $\begin{array}{l}\text { B-57 } \\
\text { B-137 } \\
\text { B-59 } \\
\text { B-91 } \\
\text { B-40 }\end{array}$ & $\begin{array}{l}\text { anfibia } \\
\text { anfibia } \\
\text { anfibia } \\
\text { anfibia } \\
\text { anfibia }\end{array}$ \\
\hline
\end{tabular}




\begin{tabular}{|c|c|c|c|}
\hline Família & Nome científico & $\begin{array}{l}\mathbf{N}^{\mathbf{o}} \\
\text { coletor }\end{array}$ & $\begin{array}{l}\text { Forma } \\
\text { de vida }\end{array}$ \\
\hline & $\begin{array}{l}\text { Paspalum millegrana Schrad. }{ }^{3} \\
\text { Paspalum pumilum Nees }\end{array}$ & $\begin{array}{l}\text { B-90 } \\
\text { B-58 }\end{array}$ & $\begin{array}{l}\text { emergente } \\
\text { anfibia }\end{array}$ \\
\hline Polygalaceae & $\begin{array}{l}\text { Polygala tenella } \text { Willd. }{ }^{3} \\
\text { Polygala sp. }\end{array}$ & $\begin{array}{l}\text { B-9 } \\
\text { B-23 }\end{array}$ & $\begin{array}{l}\text { anfibia } \\
\text { emergente }\end{array}$ \\
\hline Rubiaceae & $\begin{array}{l}\text { Borreria capitata } \text { DC. }^{2} \\
\text { Spermacoce verticillata } \mathrm{L}^{2} \\
\text { Coccocypselum capitatum }(\mathrm{Graham}) \text { C.B.Costa \& Mamede } \\
\text { Oldenlandia salzmannii }(\mathrm{DC} .) \text { Benth. \& Hook.f. ex B.D.Jacks. } \\
\text { Melanopsidium nigrum Colla }{ }^{1} \\
\text { Mitracarpum sellowianum } \text { Cham. \& Schltdl. } \\
\text { Perama hirsuta Aubl. } \\
\text { Tocoyena bullata } \text { (Vell.) Mart. }\end{array}$ & $\begin{array}{l}\text { B-27 } \\
\text { B-10 } \\
\text { B-44 } \\
\text { B-14 } \\
\text { B-32 } \\
\text { B-12 } \\
\text { B-29 } \\
\text { B-70 }\end{array}$ & $\begin{array}{l}\text { emergente } \\
\text { emergente } \\
\text { anfibia } \\
\text { anfibia } \\
\text { emergente } \\
\text { emergente } \\
\text { anfibia } \\
\text { arbustiva }\end{array}$ \\
\hline Sapindaceae & $\begin{array}{l}\text { Dodonaea viscosa } \text { Jacq. }^{3} \\
\text { Paullinia weinmanniifolia A.Gray }\end{array}$ & $\begin{array}{l}\text { B-102 } \\
\text { B-34 }\end{array}$ & $\begin{array}{l}\text { arbustiva } \\
\text { escandente }\end{array}$ \\
\hline Sapotaceae & Manilkara subsericea (Mart.) Dubard & B-52 & arbustiva \\
\hline Scrophulariaceae & $\begin{array}{l}\text { Esterhazya splendida J.C.Mikan. } \\
\text { Scoparia dulcis L. }\end{array}$ & $\begin{array}{l}\text { B- } 48 \\
\text { B-68 }\end{array}$ & $\begin{array}{l}\text { anfibia } \\
\text { emergente }\end{array}$ \\
\hline Smilacaceae & Smilax rufescens Griseb. & B-7 & emergente \\
\hline Solanaceae & Solanum cordifolium Dunal $^{3}$ & B-130 & emergente \\
\hline Turneraceae & $\begin{array}{l}\text { Turnera sp. } \\
\text { Turnera lucida Urban }\end{array}$ & $\begin{array}{l}\text { B-106 } \\
\text { B-5 }\end{array}$ & $\begin{array}{l}\text { anfibia } \\
\text { anfibia }\end{array}$ \\
\hline Typhaceae & Typha angustifolia $\mathrm{L} .^{2}$ & B-2 & emergente \\
\hline Verbenaceae & $\begin{array}{l}\text { Lantana camara } \text { L. }^{2} \\
\text { Lippia } \text { sp. } 1 \\
\text { Lippia } \text { sp. } 2 \\
\text { Stachytarpheta schottiana Schauer } \\
\text { Stachytarpheta } \text { sp. }\end{array}$ & $\begin{array}{l}\text { B-21 } \\
\text { B-22 } \\
\text { B-66 } \\
\text { B-54 } \\
\text { B-73 }\end{array}$ & $\begin{array}{l}\text { emergente } \\
\text { emergente } \\
\text { anfibia } \\
\text { anfibia } \\
\text { anfibia }\end{array}$ \\
\hline Violaceae & Hybanthus calceolaria $($ L.) Oken & B-89 & anfibia \\
\hline Xyridaceae & Xyris jupicai Rich. & B-8 & emergente \\
\hline $\begin{array}{l}\text { Lycophytas } \\
\text { Lycopodiaceae }\end{array}$ & Lycopodiella prostrata (R.M. Harper) Cranfill & B-77 & emergente \\
\hline Monilophytas & & & \\
\hline Dennstaedtiaceae & Lindsaea stricta (Sw.) Dryand. & B-16 & emergente \\
\hline Gleicheniaceae & Dicranopteris flexuosa (Schrad.) Underw. & B-78 & anfibia \\
\hline Lygodiaceae & Lygodium volubile $\mathrm{Sw}$. & B-31 & escandente \\
\hline Pteridaceae & Acrostichum danaeifolium (Fée) C.Presl & B-88 & emergente \\
\hline Salviniaceae & Salvinia auriculata Aubl. ${ }^{2}$ & B-55 & flutuante-livre \\
\hline
\end{tabular}

Para a planície costeira do estado do Rio de Janeiro são listadas 113 espécies de Angiospermas distribuídas em 40 famílias (Bove et al. 2003). Destas, a família Cyperaceae alcança maior riqueza com 23 espécies, Fabaceae e Onagraceae aparecem com sete espécies cada, seguidas por Poaceae com seis espécies e Asteraceae e Scrophulariaceae com cinco. Em outros trabalhos realizados para este tipo de ambiente (Irgang \& Gastal Junior 1996; Matias et al. 2003; Das Neves 2006; Amaral et al. 2008), a família Cyperaceae também aparece com maior riqueza dentre as demais famílias, fato este 
justificado por Matias et al. (2003) como sendo resultado do eficaz sistema de rizomas subterrâneos que confere aos membros da família maior eficiência vegetativa e dominância competitiva.

Apesar de Cyperaceae estar entre as famílias mais representativas no presente estudo, Asteraceae foi a família dominante em número de espécies com 13 táxons. Kita \& Souza (2003) encontraram Poaceae com dominância florística em uma planície alagável do Paraná, enquanto que Lisbôa \& Gastal Junior (2003) apontam Fabaceae como sendo a família mais representativa em um lago no Rio Grande do Sul. Estes últimos autores apontam a ocorrência de espécies exóticas provenientes de outros ambientes na área de estudo, sendo este tipo de pressão antrópica uma possível justificativa para a alternância de famílias já que atualmente a segunda maior ameaça a biodiversidade é atribuída à introdução de espécies exóticas invasoras (Ziller 2006).

Algumas espécies presentes na formação brejo-herbáceo de Morada do Sol ocorrem em outras formações de restinga como formação florestal não inundável: Byrsonima sericea, Calyptranthes brasiliensis, Guapira opposita, Lundia cordata, Melanopsidium nigrum, Manilkara subsericea, Garcinia brasiliensis, entre outras (Fabris \& César 1996; Pereira \& Zambom 1998; Pereira et al. 2000; Assis et al. 2004), e arbustiva aberta inundável: Cuphea flava, Chamaecrista ramosa, Cereus fernambucensis, Marcetia taxifolia e Senna apendiculata (Pereira \& Araújo 1995). A formação arbustiva aberta não inundável esteve representada através de Oxypetalum alpinum, Pera glabrata e Stylosanthes viscosa (Colodete \& Pereira 2007). Outras espécies como Maytenus obtusifolia, Paullinia weinmanniifolia, Smilax rufescens são comuns em formação arbustiva fechada não inundável (Fabris \& Pereira 1998).

A espécie Melanopsidium nigrum encontrase incluída pelo IBAMA (2008) na lista de espécies da flora brasileira ameaçadas de extinção.

Pereira \& Simonelli (1996) estudando o uso da vegetação de restinga pelos Tupinikim em uma Reserva Indígena no norte do Espírito Santo detectaram 26 espécies com potencial de utilização. Destas, Schinus terebinthifolius, Cereus fernambucensis, Garcinia brasiliensis, Passiflora alata e Smilax rufescens podem ser encontradas também no brejo-herbáceo de Morada do Sol indicando o importante valor etnobiológico que as espécies presentes nesta formação possuem.
Constatou-se a ocorrência de 28 espécies daninhas ou invasoras (Tab. 1), estas apresentam grande potencial de adaptação e reprodução a ponto de substituir espécies nativas e alterar os processos ecológicos naturais (Ziller 2006). Verificou-se inúmeros pontos de extração ilegal de areia e testemunhos de queimadas na restinga de Morada do Sol, sendo estes fatores de perturbação potenciais para propiciar condições favoráveis para entrada destas espécies.

A conservação destes ecossistemas dependerá de estudos mais acentuados para melhor conhecimento de seus aspectos florísticos no estado do Espírito Santo. Vale ressaltar que a ocorrência de espécies anuais neste tipo de ecossistema é reflexo da sazonalidade do regime hídrico e estudos com esforço amostral superiores a um ano poderiam evidenciar uma maior representatividade florística.

\section{Agradecimentos}

Os autores gostariam de agradecer o precioso apoio prestado por Diogo Andrade Koski nas expedições e aos técnicos e estagiários do Herbário VIES o auxílio e orientações prestados durante o depósito do material botânico.

\section{Referências}

Amaral, M.C.E.; Bittrich, V.; Anderson, L.O. \& Aona, L.Y. 2008. Guia de campo para plantas aquáticas e palustres do estado de São Paulo. Holos/FAPESP, Ribeirão Preto. 452p.

APG III - Angiosperm Phylogeny Group. 2009. An update of the Angiosperm Phylogeny Group classification for the orders and families of flowering plants: APG III. Botanic Journal Linnean Society 161: 105-121.

Assis, A.M.; Thomaz, L.D. \& Pereira, O.J. 2004. Florística de um trecho de floresta de restinga no município de Guarapari, Espírito Santo, Brasil. Acta Botanica Brasilica 18: 191-201.

Barroso, G.M. 1991a. Sistemática de angiospermas do Brasil. Vol. 2. UFV, Viçosa. 377p.

Barroso, G.M. 1991b. Sistemática de angiospermas do Brasil. Vol. 3. UFV, Viçosa. 326p.

Barroso, G.M.; Peixoto, A.L.; Ichaso, C.L.F.; Guimarães, E.F. \& Costa C.G. 2002. Sistemática de angiospermas do Brasil. $2^{\mathrm{a}}$ ed. Vol. 1. UFV, Viçosa. 309p.

Bove, C.P.; Gil, A.S.B.; Moreira, C.B. \& Dos Anjos, R.F.B. 2003. Hidrófitas fanerogâmicas de ecossistemas aquáticos temporários da planície costeira do estado do Rio de Janeiro, Brasil. Acta Botanica Brasílica 17: 119-135. 
Colodete, M.F. \& Pereira, O.J. 2007. Levantamento floristico da restinga de Regência, Linhares/ES. Revista Brasileira de Biociências 5: 558-560.

Christenhusz, M.J.M; Zhang, X. \& Schneider, H. 2011. A linear sequence of extant families and genera of lycophytas and ferns. Phytotaxa 19: 7-54.

Das Neves, E.L.; Leite, K.R.B.; França, F. \& Melo, E. 2006. Plantas aquáticas vasculares em uma lagoa de planície costeira no município de Candeias, Bahia, Brasil. Sitientibus: Série Ciências Biologicas 6: 24-29.

Fabris, L.C. \& César, O. 1996. Estudos florísticos em uma mata litorânea no sul do Estado do Espírito Santo. Boletim do Museu de Biologia Mello Leitão 5: 15-46.

Fabris, L.C. \& Pereira, O.J. 1998. Florística da formação pós-praia: na restinga de Setiba, Guarapari (ES). In: Watanabe, S. (org.). Anais do simpósio de ecossistemas brasileiros. Vol. 3. ACIESP, São Paulo. Pp. 165-176.

Fidalgo, O. \& Bononi, V.L. 1989. Técnicas de coleta, preservação e herborização de material botânico. Instituto de Botânica, São Paulo. 62p.

IBAMA. Lista Oficial de Espécies da Flora Brasileira Ameaçada de Extinção. Instrução Normativa n ${ }^{\circ}$ 177, 18 de junho de 2008.

Irgang, B.E. \& Gastal Junior, C.V.S. 1996. Macrófitas aquáticas da planície costeira do RS. UFRGS, Porto Alegre. 290p.

Kita, K.K. \& Souza, M.C. 2003. Levantamento florístico e fitofisionomia da lagoa Figueira e seu entorno na planície alagável do alto rio Paraná, Porto Rico, estado do Paraná, Brasil. Acta Scientiarum: Biological Sciences 25: 145-155.

Lacerda, L.D.; Araújo, D.S.D. \& Maciel, N.C. 1993. Dry coastal ecossystems of the tropical Brazilian coast. In: Marel, E. Van Der. (ed.). Dry coastal ecossystems of the word. Elsevier, Amsterdan. Pp. 477-493.

Lacerda, L.D.; Cunha, C.T. \& Seeliger, U. 1986. Distribuição de nutrientes em perfis de sedimentos em brejos costeiros tropicais e temperados. Acta Limnologica Brasiliensis 1: 387-399.

Lisbôa, F.F. \& Gastal-Júnior, C.V.S. 2003. Levantamento das macrófitas aquáticas na beira do Lago Guaíba no município de Guaíba, RS/Brasil. Caderno de Pesquisa Série Biologia, Santa Cruz do Sul 15: 17-27.

Lorenzi, H. 2008. Plantas daninhas do Brasil: terrestres, aquáticas, parasitas e tóxicas. $4^{\mathrm{a}}$ ed. Plantarum, Nova Odessa. 640p.

Magnago, L.F.S.; Pereira, O.J.; Matos, F.A.R. \& Souza, P.F. 2007. Caracterização fitofisionômica da Restinga na Morada do Sol, Vila Velha/ES. Revista Brasileira de Biociências 5: 456-458.
Martins, M.L.L.; Luceño, M. \& Carvalho-Okano, R.M. 1999. Cyperaceae do Parque Estadual Paulo Cesar Vinha, Guarapari, ES, Brasil. Acta Botanica Brasilica 13: 187-222.

Matias, L.Q.; Amado, E.R. \& Nunes, E.P. 2003. Macrófitas aquáticas da Lagoa de Jijoca de Jericoacoara, Ceará, Brasil. Acta Botanica Brasilica 17: 623-631.

Pedralli, G. 1990. Macrófitos aquáticos: técnicas e métodos de estudos. Estudos de Biologia 26: 5-24.

Pereira, O.J. 2003. Restinga: origem, estrutura e diversidade. In: Jardim M.A.G.; Bastos, M.N.C \& Santos, J.U.M. (orgs.). Desafios da botânica brasileira no novo milênio: inventário, sistematização e conservação da biodiversidade vegetal. Vol. 1. Sociedade Botânica do Brasil/Universidade Federal Rural da Amazônia/ Museu Paraense Emílio Goeldi, Belém. Pp. 177-179.

Pereira, O.J. \& Araújo, D.S.D. 1995. Estrutura da vegetação de entre moitas da formação aberta de Ericaceae no Parque Estadual de Setiba, ES. In: Esteves, F.A. (ed.). Oecologia brasiliensis: estrutura, funcionamento e manejo de ecossistemas brasileiros. Vol. 1. Vitória. Pp. 245-257.

Pereira, O.J. \& Araújo, D.S.D. 2000. Análise florística das restingas dos estados do Espírito Santo e Rio de Janeiro. In: Esteves, F.A. \& Lacerda, L.D. (eds.). Ecologia de lagoas costeiras. NUPEM/UFRJ, Macaé. Pp. 25-63.

Pereira, O.J. \& Simonelli, M. 1996. Vegetação de restinga e os índios Tupinikim da Reserva Indígena de Comboios - Aracruz, ES. I - Plantas utilizadas na alimentação, caça, confecção de artefatos e tinturas. Cadernos de Pesquisas da UFES 6: 92-106.

Pereira, O.J. \& Zambom, O. 1998. Composição florística da restinga de Interlagos, Vila Velha (ES). In: Watanabe, S. (org.). Anais do IV simpósio de ecossistemas brasileiros. Vol. 3. ACIESP, São Paulo. Pp. 129-139.

Pereira, O.J.; Borgo, J.H.; Rodrigues, I.D. \& Assis, A.M. 2000. Composição florística de uma floresta de restinga no município da Serra-ES. In: Watanabe, S. (org.). Anais do V simpósio de ecossistemas costeiros: conservação. Vol. 3. ACIESP, Vitória. P. 109.

Souza, V.C. \& Lorenzi, H. 2005. Botânica sistemática: guia ilustrado para identificação das famílias de Angiospermas da flora brasileira, baseado em APG II. Instituto Plantarum, São Paulo. 639p.

Ziller, S.R. 2006. Espécies exóticas da flora invasoras em Unidades de Conservação. In: Campos, J.B.; Tossulino, M.G.P. \& Müller, C.R.C. (eds.). Unidades de Conservação: ações para valorização da biodiversidade. Instituto Ambiental do Paraná, Curitiba. Pp. 34-52. 